\title{
Simplificação da equação de viabilidade para predizer a longevidade de sementes de milho e soja
}

\author{
Claudinei Andreoli(1)
}

(1)Embrapa Soja, Caixa Postal 231, CEP 86060-970 Londrina, PR. E-mail: andreoli@cnpso.embrapa.br

Resumo - A equação de Ellis \& Roberts utiliza a temperatura, a umidade e a qualidade inicial da semente para predizer sua longevidade, porém exige experimentos complexos e demorados. O objetivo deste trabalho foi simplificar a equação de viabilidade para predizer a longevidade da semente de milho e soja em condições de armazenamento aberto. A equação simplificada é explicada pelo modelo $\mathrm{Vp}=\mathrm{Vi}$ - $(\operatorname{tg} \beta)$.p em que $\mathrm{Vp}$ é a viabilidade em probit no período $\mathrm{p}$, Vi é a germinação inicial do lote e tg $\beta$ é a taxa de deterioração da semente para cada espécie. Sementes de milho BRS201 e BRS206 e soja cultivar IAC-8 e MG/BR 46 (Conquista) foram embaladas em sacos de papel e armazenadas por 0, 30, 60, 90, 120, 150, 180, 240, 300 e 360 dias, em galpões abertos, em Sete Lagoas, MG, e Brasília, DF. Os dados foram transformados em 'probit' e a declividade da reta $(\operatorname{tg} \beta)$ foi calculada entre 0 e 30 dias. $O$ coeficiente $(\operatorname{tg} \beta)$ variou de $1,4767.10^{-3}$ a $2,687.10^{-3}$ em milho e de $2,868.10^{-3}$ a $3,617.10^{-3}$ em soja, dependendo das condições climáticas do armazém. A germinação da semente de soja declinou mais rapidamente que a de milho. $\mathrm{O}$ modelo prediz com precisão a longevidade das sementes de milho e soja em armazém aberto.

Termos para indexação: germinação, armazenamento, vigor, deterioração.

\section{Simplification of viability equation to predict seed longevity of corn and soybean}

Abstract - Temperature, moisture and initial quality are important factors in Ellis and Roberts' equation to predict seed longevity, however it relies on complex experiments of seed aging. The objective of this work was to simplify the equation for predicting changes in seed viability during open warehouse storage. The simplified equation proposed is given by the model $\mathrm{Vp}=\mathrm{Vi}-(\operatorname{tg} \beta)$, in which $\mathrm{Vp}$ is seed viability in probit for a period $\mathrm{p}$ (days), $\mathrm{Vi}$ is the initial seed viability in probit and $\operatorname{tg} \beta$ is the seed deterioration rate for each specie at determined storage conditions. Seeds of hybrid corn BRS201 and BRS206 and soybean varieties IAC-8 and MG/BR 46 (Conquista) were packed in multiwall paper bags and stored for 0, 30, 60, 90, 120, 150, 180, 240, 300 and 360 days in open warehouse at two locations, Sete Lagoas, Minas Gerais State and Brasília, Federal District. The data were transformed in probit and the deterioration rate $(\operatorname{tg} \beta)$ between 0 and 30 days was calculated. The coefficient $\operatorname{tg} \beta$ ranged from $1.476 \times 10^{-3}$ to $2.687 \times 10^{-3}$ for corn and from $2.868 \times 10^{-3}$ to $3.617 \times 10^{-3}$ for soybean, depending on locations. These data indicate that soybean seed germination declined faster than corn. This model has accurately predicted corn and soybean seed germination after an open storage period.

Index terms: germination, storage, vigor, aging.

\section{Introdução}

A temperatura, a umidade relativa do ar e a qualidade inicial influenciam a perda de vigor no armazenamento (Roberts, 1986). A rápida deterioração da semente de soja durante o armazenamento é afetada pela umidade e temperatura (Burris, 1980).

Os produtores, não conhecendo a curva de viabilidade, descartam muitos lotes de sementes que ainda possuem qualidade após o beneficiamento, o ensacamento e o armazenamento. A determinação da qualidade da semente, germinação e vigor na colheita e no bene- ficiamento não é suficiente para estimar as mudanças da qualidade, durante o armazenamento. Portanto, para predizer a longevidade da semente em condições controladas de temperatura e umidade da semente, a equação básica de viabilidade foi descrita quanto ao trigo e arroz (Roberts, 1960, 1961) e sua aplicação se estendeu a um grande número de espécies (Roberts, 1973).

A fim de melhorar a confiabilidade da equação, Ellis \& Roberts (1980) propuseram a versão melhorada (2) na equação básica (1), a seguir:

$\log \mathrm{p}_{50}=\mathrm{K}_{\mathrm{v}}-\mathrm{C}_{1} \mathrm{~m}-\mathrm{C}_{2} \mathrm{t}$, 
$\mathrm{v}=\mathrm{Ki}-\mathrm{p} / 10^{\mathrm{K}} \mathrm{e}_{\mathrm{W}} \mathrm{C}_{\mathrm{W}} \log \mathrm{m}-\mathrm{C}_{\mathrm{H}} \mathrm{t}-\mathrm{C}_{\mathrm{Q}} \mathrm{t}^{2}$,

em que v é a viabilidade de sementes em 'probit' em qualquer período $\mathrm{p}$, para a combinação de temperatura (t) e umidade (m); as constantes, $\mathrm{K}_{\mathrm{e}}, \mathrm{C}_{\mathrm{w}}, \mathrm{C}_{\mathrm{H}}$ e $\mathrm{C}_{\mathrm{Q}}$ são específicas para cada espécie, independentemente do genótipo e da qualidade inicial da semente; $\mathrm{K}_{\mathrm{i}}$ é específico do lote de semente e é uma medida da sua qualidade inicial. Na prática, a equação (2) apresenta cálculos matemáticos difíceis e a determinação das constantes exige experimentos complexos e demorados.

Sementes de milho e soja armazenadas em galpões abertos, normalmente não estão expostas à temperatura ou umidade relativa constantes. Por isso, Delouche \& Baskin (1973) propuseram o teste de envelhecimento precoce para predizer o potencial de armazenamento de lotes de sementes de várias espécies em condições de ambiente aberto, mas não desenvolveram um modelo. Tekrony et al. (1993), utilizando as equações de viabilidade de Ellis \& Roberts (1980), propuseram um protocolo para predizer a germinação de sementes de soja, em armazém aberto. Todavia, esse modelo somente pode ser aplicado se a temperatura no armazém e a umidade da semente forem determinadas diariamente. Predições exatas da deterioração da semente sob mudanças climáticas no armazém, portanto, são de grande valia para os produtores de sementes.

O objetivo deste trabalho foi simplificar a equação básica de viabilidade para predizer as mudanças na germinação e longevidade da semente de milho e soja, durante o armazenamento aberto, bem como testar o modelo proposto em diferentes localidades.

\section{Material e Métodos}

\section{Equação simplificada}

A relação entre a temperatura, a umidade da semente e o período médio de viabilidade foi proposta po Roberts $(1960,1961)$ e Roberts \& Abdalla (1968). Assim, a perda de viabilidade foi definida por três equações básicas de viabilidade (Roberts, 1972), a saber: 1) A freqüência de sementes mortas num determinado período, em uma população armazenada em condições constantes, é descrita pela distribuição normal:

$y=\frac{1}{\sigma \sqrt{2 \pi}} e^{\frac{(p-\mu)^{2}}{2 \sigma^{2}}}$,

em que y é a freqüência relativa de sementes mortas no tempo $\mathrm{p} ; \mu$ é o período de viabilidade média $\left(\mathrm{p}_{50}\right) ; \sigma$ é desvio-padrão da distribuição das sementes mortas no tempo; 2) A dispersão da distribuição no tempo é proporcional ao período de viabilidade média,

$$
\sigma=\mathrm{K}_{\sigma} \cdot \mathrm{p}_{50},
$$

em que $\mathrm{K}_{\sigma}$ é uma constante das espécies; 3) A relação entre temperatura, grau de umidade da semente e período de viabilidade médio é descrita pela seguinte equação:

$\log \mathrm{p}_{50}=\mathrm{K}_{\mathrm{v}}-\mathrm{C}_{1} \mathrm{~m}-\mathrm{C}_{2} \mathrm{t}$,

em que m é a umidade da semente (por cento, em base úmida); t é a temperatura $\left({ }^{\circ} \mathrm{C}\right) ; \mathrm{K}_{\mathrm{v}}, \mathrm{C}_{1}$ e $\mathrm{C}_{2}$ são constantes.

Conforme a equação (3), as curvas de sobrevivência da semente são retas de coeficiente de regressão negativo, quando a porcentagem de viabilidade é transformada em 'probit'. Nessas retas, o coeficiente de regressão é dado por 1/ $\sigma$ (Finney, 1971; Ellis \& Roberts, 1980), em que $\sigma$ é o desvio-padrão da distribuição de sementes mortas no tempo. Assim, a equação de viabilidade poder ser descrita como:

$\mathrm{v}=\mathrm{K}_{\mathrm{i}}-(1 / \sigma) \cdot \mathrm{p}$,

em que v é a germinação em probit no tempo $\mathrm{p} ; \mathrm{K}_{\mathrm{i}}=\mathrm{v}$ quando $\mathrm{p}=0$.

Com base na Figura 1, tem-se:

$\frac{\mathrm{Vp}-\mathrm{Vi}}{\mathrm{p}}=-(\operatorname{tg} \beta)$.

Portanto, o coeficiente de regressão da equação melhorada (2), $\sigma$, dado pelo termo $10 \mathrm{~K}_{\mathrm{e}}-\mathrm{C}_{\mathrm{w}} \operatorname{logm}-\mathrm{C}_{\mathrm{H}} \mathrm{t}-\mathrm{C}_{\mathrm{Q}} \mathrm{t}^{2}$, é igual ao coeficiente $\operatorname{tg} \beta$ da equação simplificada (7), e substituindo em (6) tem-se a equação simplificada: $\mathrm{Vp}=\mathrm{Vi}-(\operatorname{tg} \beta) \cdot \mathrm{p}$,

em que $\mathrm{Vp}$ é a viabilidade das sementes em 'probit' no tempo p; Vi é a germinação inicial em 'probit'; $\operatorname{tg} \beta$ é a taxa de deterioração da semente para cada espécie (Figura 1). A constante $\operatorname{tg} \beta$ é específica de cada espécie e das condições ambientais do armazém, mas independe do genótipo e da qualidade inicial da semente (Roberts \& Abdalla, 1968; Roberts, 1973). Vi é específico a cada lote de sementes e é medida da sua qualidade inicial. O valor de (-tg $\beta)$ é estimado, calculando-se o declínio da viabilidade (Vp - Vi) por um determinado período $(\Delta \mathrm{p})$, nas condições específicas do armazém.

Desde que Vi seja, de fato, a porcentagem de viabilidade em 'probit' no início do armazenamento, é possível estimar seu valor por meio de um teste de germinação no início do armazenamento. Roberts (1986) argumen- 
tou que o simples teste de germinação não estima adequadamente a qualidade da semente no início do armazenamento. $\mathrm{O}$ erro de amostragem no teste de germinação com 400 sementes pode ser alto e, consequientemente, o intervalo de confiança desse valor também pode ser elevado. Uma pequena diferença na porcentagem de germinação para altos valores pode causar grandes efeitos no valor de Vi também, no potencial de longevidade.

\section{Análises do material e estatística}

Para testar o modelo proposto na equação (8), sementes de milho híbrido BRS201 e BRS206, e de soja cultivar IAC-8 e MG/BR 46 (Conquista) foram emba-

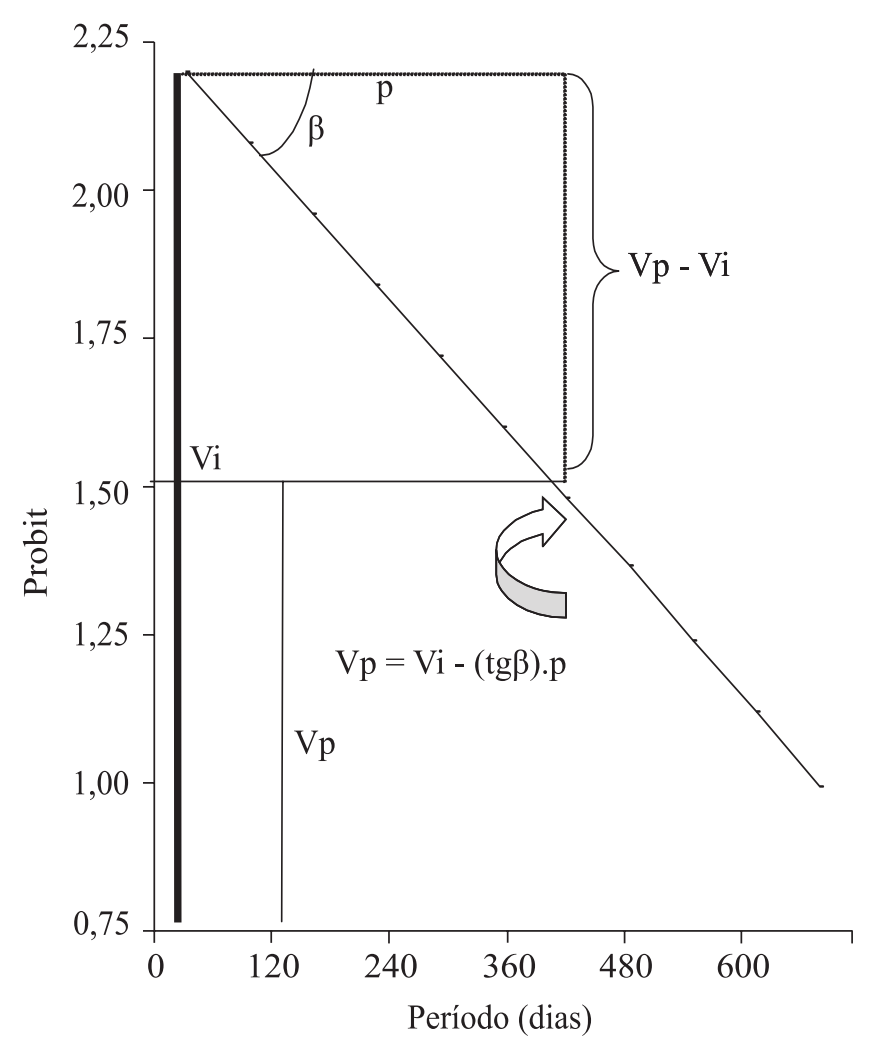

Figura 1. Valores da porcentagem de viabilidade são transformados em 'probit', representam que a curva de sobrevivência é uma reta de coeficiente de regressão $1 / \sigma$, onde $\sigma$ é o desvio-padrão da distribuição e o modelo simplificado pode ser descrito por $\left.\left(\mathrm{V}_{\mathrm{p}}-\mathrm{V}_{\mathrm{i}}\right) / \mathrm{p}=-\operatorname{tg} \beta\right)$ e, portanto, por $\mathrm{Vp}=\mathrm{Vi}$ $(\operatorname{tg} \beta)$. p, em que $V p$ é a viabilidade da semente em 'probit' no tempo $\mathrm{p}$, Vi é a germinação inicial em 'probit', $\mathrm{Vp}=\mathrm{Vi}$ quando $\mathrm{p}=0$, e $\operatorname{tg} \beta$ é a taxa de deterioração da semente para cada espécie. ladas em sacos de papel duplo (multifoliado), comumente utilizados pelas empresas sementeiras, e armazenadas em galpão aberto, em Sete Lagoas, MG e Brasília, DF, por $0,30,60,90,120,180,240,300$ e 360 dias. Em cada período, amostras foram coletadas para análise de germinação e grau de umidade. No início de cada armazenamento (julho de 1996), a qualidade inicial das sementes (Vi) foi determinada (Tabela 1). Durante o período de armazenamento, a temperatura e a umidade relativa média foram registradas.

A germinação e o grau de umidade das sementes foram analisados de acordo com as Regras para Análise de Sementes (Brasil, 1992). A germinação das sementes de milho e soja foi conduzida em rolos de papel toalha Germitest, com oito repetições de 50 sementes para cada período de armazenamento. O grau de umidade das sementes foi determinado pelo método de estufa a $105^{\circ} \mathrm{C}$, por 24 horas, utilizando-se quatro repetições de $25 \mathrm{~g}$ para cada amostra e os resultados foram expressos em g de água por $\mathrm{kg}$ de semente.

Os dados de germinação foram transformados em 'probit' e ajustados pela equação (8), para predizer a viabilidade das sementes de milho e soja durante o armazenamento. Na determinação do coeficiente angu$\operatorname{lar} \operatorname{tg} \beta$ (taxa de deterioração da semente) em cada localidade e em cada espécie, utilizou-se a viabilidade inicial e após 30 dias $\left(\mathrm{Vi}-\mathrm{V}_{30}\right)$. A seguir, a equação simplificada foi usada para predizer a germinação da semente durante cada período de armazenamento.

Tabela 1. Germinação inicial média (Vi), viabilidade em relação ao período médio de 180 dias $\left(\mathrm{p}_{180}\right)$ e coeficientes de determinação $\left(r^{2}\right)$ observados em sementes de milho e soja armazenadas em duas localidades.

\begin{tabular}{lcccc}
\hline Genótipo & Localidade & \multicolumn{2}{c}{ Germinação (\%) } & \multirow{2}{*}{ r $^{2}$} \\
\cline { 3 - 4 } & & Vi & $\mathrm{p}_{180}$ & \\
\hline \multirow{2}{*}{ BRS 201 } & Sete Lagoas, MG & Milho & & \\
BRS 206 & Sete Lagoas, MG & 96 & 96,4 & 0,97 \\
BRS 201 & Brasília, DF & 92 & 84,2 & 0,96 \\
BRS 206 & Brasília, DF & 98 & 94,6 & 0,96 \\
\hline & & Soja & & \\
IAC 8 & Sete Lagoas, MG & 90 & 69,0 & 0,95 \\
IAC 8 & Brasília, DF & 90 & 78,0 & 0,98 \\
\hline
\end{tabular}

Pesq. agropec. bras., Brasília, v.39, n.9, p.911-917, set. 2004 
Os dados de cada localidade, para cada espécie, foram ajustados pelo modelo da equação simplificada e os valores de $\mathrm{r}^{2}$, entre os valores observados e esperados, foram calculados para estimar o grau de confiabilidade do modelo proposto.

\section{Resultados e Discussão}

O teor de água na semente de milho variou de $95 \mathrm{~g}$ no período seco (maio a julho) a $110 \mathrm{~g}$ de água/kg de semente, no período chuvoso (novembro a fevereiro).

A germinação predita pela equação simplificada (equação 8) foi comparada com a germinação observada no período de 0 a 360 dias para milho BRS201 e BRS206, nas duas localidades (Figura 2). A equação simplificada previu com precisão a queda de viabilidade do lote de semente do BRS201 e também a germinação acima de $81 \%$ após 360 dias de armazenamento, nas duas localidades. A germinação do BRS206 permaneceu acima de $86 \%$, após 360 dias de armazenamento. A queda de viabilidade foi mais rápida para o BRS201, em Brasília, DF, no período de 60 a 180 dias. O modelo prognosticou a queda de viabilidade para os dois híbridos de milho nas duas localidades, entretanto a diferença entre os valores observados e os calculados foi menor que $2 \%$ no BRS201 e que $4 \%$ no BRS206, no final do armazenamento. As altas correlações entre os valores observados e estimados pela equação $\left(r^{2}>0,96\right)$ revelam a alta confiabilidade do modelo em predizer a qualidade da semente no armazenamento em galpão aberto.

A taxa de deterioração da semente, estimada pelo coeficiente angular $(\operatorname{tg} \beta)$, variou de 0,0018433 a 0,001476, nos híbridos BRS206 e BRS201, respectivamente (Figura 2). Esses valores indicam que a taxa de deterioração em Sete Lagoas, MG, foi maior do que em
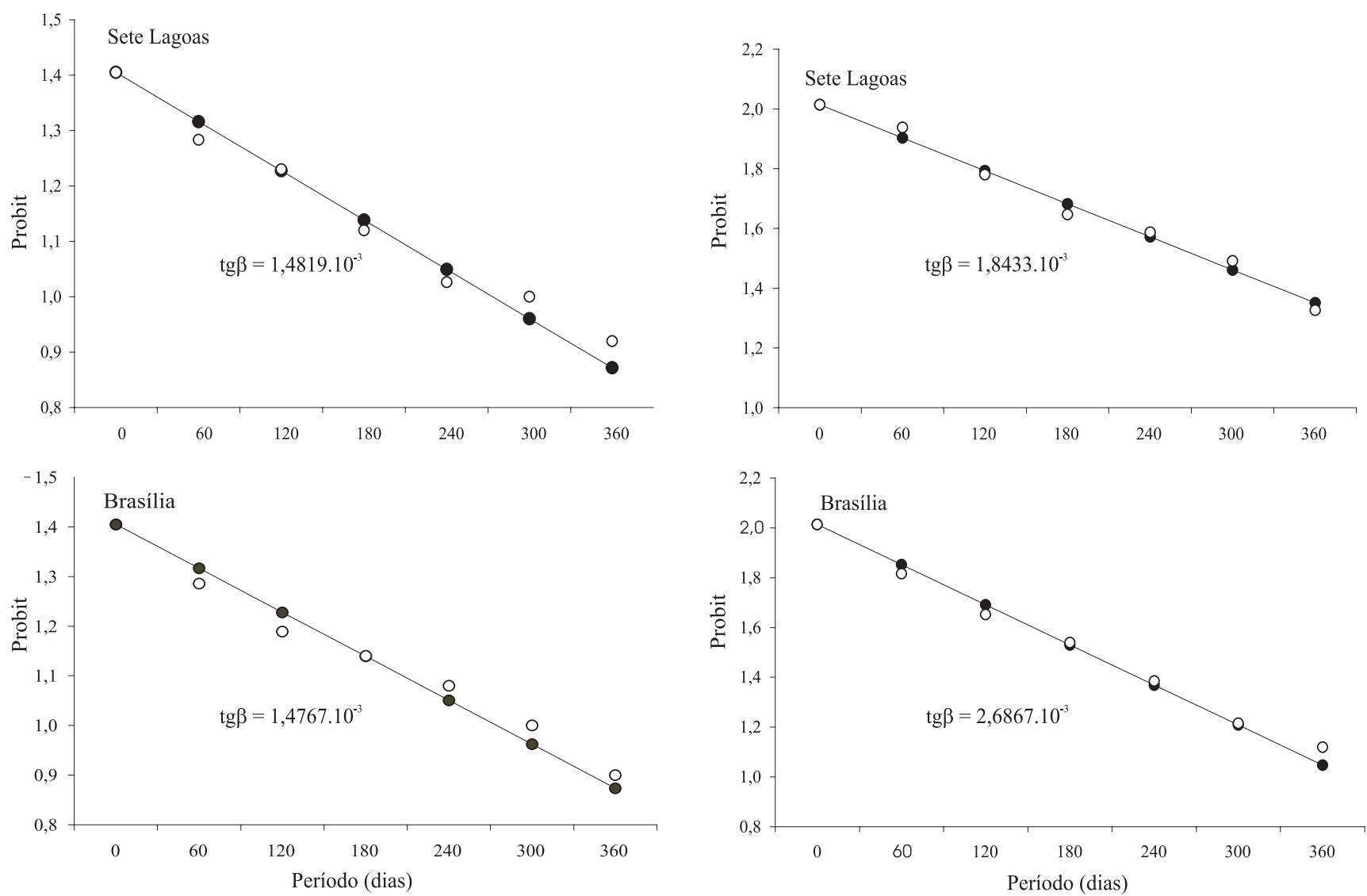

Figura 2. Germinação predita (@) pela equação simplificada e observada (O) dos lotes de semente de milho BRS201 e BRS 206, armazenados em Sete Lagoas, MG, e em Brasília, DF, a partir de julho de 1996. 
Brasília, DF. Observa-se que o ângulo ( $\beta$ ) é influenciado pelas condições climáticas locais, pois quanto mais quente e úmido o ambiente maior será a taxa de deterioração da semente (maior o ângulo $\beta$ ) e, conseqüentemente, maior será a declividade da reta. De fato, o valor de $\operatorname{tg} \beta$ de soja é maior do que o de milho (Figura $2 \mathrm{e}$ 3). Coincidentemente, o modelo simplificado (Vp - Vi)/ $\mathrm{p}=-(\operatorname{tg} \beta)$ mostra que a taxa de deterioração da semente $(\operatorname{tg} \beta)$ equivale a $1 / \sigma$ da equação de Ellis \& Roberts (1980). Nem o genótipo e nem a qualidade inicial da semente afetam a declividade $(1 / \sigma$ e $\operatorname{tg} \beta)$ das curvas, mas somente a intercepção, $K_{i}$, da equação de viabilidade (2) é afetada por tais fatores (Roberts, 1973; Roberts \& Ellis, 1989). Portanto, a equação simplificada proposta pode ser descrita pelo modelo: $\mathrm{Vp}=\mathrm{Vi}-(\operatorname{tg} \beta) \cdot \mathrm{p}$, em que Vp é a viabilidade da semente em 'probit' no período $\mathrm{p}, \mathrm{Vi}=\mathrm{V} p$ quando $\mathrm{p}=0$, e Vi é a qualidade inicial da semente.
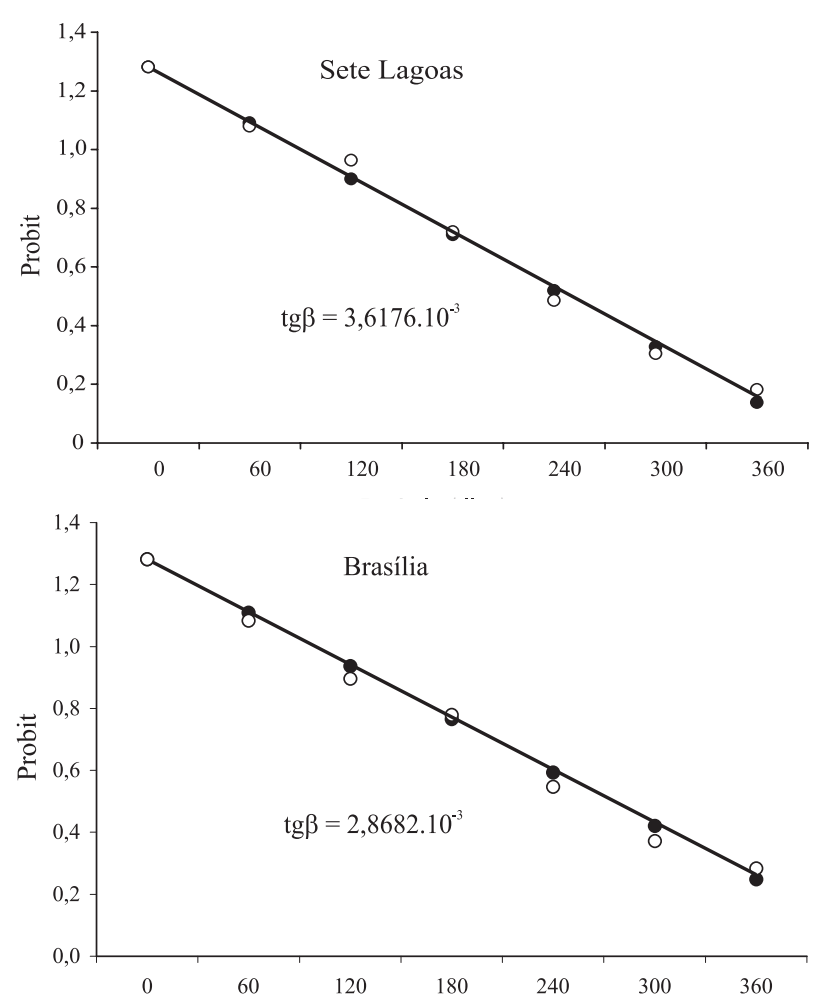

Figura 3. Germinação predita (•) pela equação simplificada e observada (O) dos lotes de semente de milho BRS206 armazenados em Sete Lagoas, MG, e em Brasília, DF, a partir de julho de 1996.
Em outro experimento controlado, sementes de milho BRS201, com germinação inicial de 93\%, foram armazenadas a $25^{\circ} \mathrm{C}$ e teor de água de $126 \mathrm{~g} / \mathrm{kg}$ de semente, condições facilmente encontradas nos armazéns dos produtores de sementes de milho nas regiões tropicais do Centro Oeste (Figura 4). A equação simplificada estimou com precisão $\left(\mathrm{r}^{2}=0,96\right)$ a queda de viabilidade de sementes de milho nessas condições. A germinação se manteve acima de $90 \%$, até os 120 dias, e declinou, rapidamente, após 180 dias, atingindo germinação abaixo de $85 \%$, valor mínimo para a comercialização de sementes.

Esses dados confirmam a confiabilidade do modelo simplificado, quando comparado com a equação de Ellis \& Roberts (1980). No entanto, a diferença fundamental entre o modelo simplificado e a equação de Ellis \& Roberts (1980) é que o modelo simplificado pode ser usado tanto em condições controladas de temperatura e umidade como em ambientes abertos. O modelo de Ellis $\&$ Roberts somente pode ser aplicado à temperatura e umidade conhecidas da semente. Outra grande vantagem do modelo simplificado é que as condições climáticas do armazém não precisam ser monitoradas, como descrito por Tekrony et al. (1993), e o produtor pode predizer a qualidade da semente em cada lote individual, necessitando apenas definir o valor de $\operatorname{tg} \beta$ para soja e milho, nas condições específicas do armazém.

O modelo simplificado é de grande valia para o produtor de sementes determinar o potencial de germina-

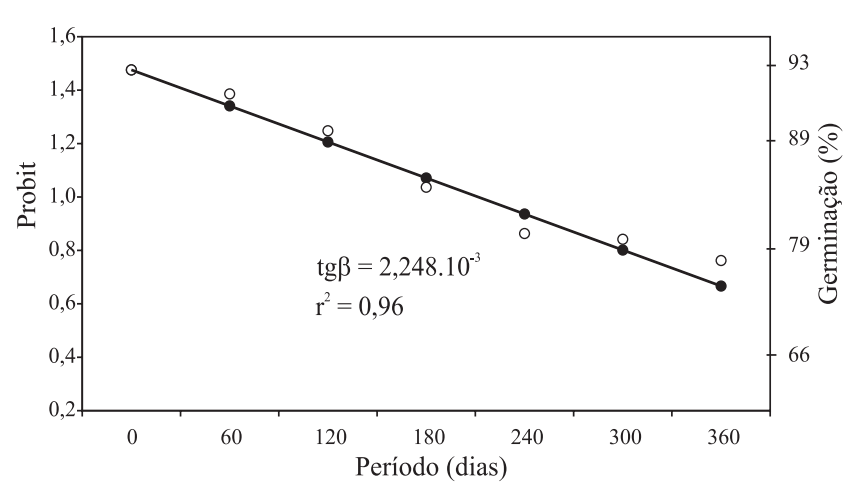

Figura 4. Germinação predita $(\bullet)$ pela equação simplificada e observada (O) do lote de semente de milho BRS201, armazenado a $25^{\circ} \mathrm{C}$ e $126 \mathrm{~g}$ de água/ $\mathrm{kg}$ de semente em Sete Lagoas, MG, a partir de abril de 1998. 
ção no momento da semeadura. Por sua vez, a equação simplificada será de grande utilidade nos programas de controle de qualidade das empresas.

A equação previu também que a germinação das sementes do BRS201 e do BRS206, armazenadas em Brasília, DF, e Sete Lagoas, MG, estava acima de $85 \%$, após nove meses de armazenamento, período que coincide com a semeadura de safrinha. Essa informação igualmente relevante para os produtores, pois sementes remanecentes da safra normal de cultivo de verão poderiam ser semeadas na safrinha.

O teor de água da semente de soja variou de $78 \mathrm{~g}$, no período chuvoso, a $117 \mathrm{~g}$ de água/ $\mathrm{kg}$ de semente, no período seco.

A germinação predita pela equação simplificada (8) foi comparada com a germinação observada no período de 30 a 360 dias de armazenamento para as sementes de soja da cultivar IAC-8, nas duas localidades (Figura 3). A equação simplificada estimou com precisão a viabilidade das sementes de soja ao longo do período de armazenamento, nas duas localidades. Como a semente está exposta diretamente às mudanças climáticas no armazém, a umidade relativa pode estar mais diretamente relacionada à atividade química da água. Assim, seria mais relevante discutir as mudanças fisiológicas da semente em termos termodinâmicos do que com base na umidade da semente, como tem sido preconizado por Vertucci \& Roos $(1990,1993)$. Isso foi comprovado por Ellis et al. (1990) que, comparando a umidade da semente com a umidade relativa de equilíbrio de amendoim, beterraba, cebola, cevada, grão-de-bico, soja, trigo e vigna, concluíram que o efeito relativo do potencial hídrico foi o mesmo para estas espécies e, provavelmente, para outras espécies ortodoxas.

Pelos valores de $\operatorname{tg} \beta$, o declínio da germinação em Sete Lagoas, MG foi mais rápido do que em Brasília DF (Figura 3). A diferença entre o valor observado e o estimado, nas duas localidades, não foi, em média, superior a $4 \%$. Os valores de $\mathrm{r}^{2}$ para Sete Lagoas, MG, e Brasília, DF, foram, respectivamente, 0,95 e 0,98 (Tabela 1).

Sementes de soja da cultivar MG/BR 46 (Conquista) com germinação inicial de $92 \%$ ('probit' = 1,4051) foram armazenadas em Sete Lagoas, MG, em 1998, para determinar o tempo, em dias, necessário para a germinação atingir $85 \%$. Pode-se afirmar com alta confiabilidade $\left(\mathrm{r}^{2}=0,94\right)$ que foram gastos 152 dias para a germinação atingir $85 \%$, período que representa o tempo entre a colheita, o processamento e a semeadura (Figura 5).



Figura 5. Comparação do tempo em (dias) que leva a germinação inicial (92\%) da soja cultivar MG/BR 46 (Conquista) decrescer para $85 \%$ durante o armazenamento, a partir de maio de 1998, nas condições de Sete Lagoas, MG, usando a equação simplificada $V p=V i-(\operatorname{tg} \beta)$. p e uma constante Vi de 1,4051 .

\section{Conclusões}

1. O modelo simplificado confirma que a semente de soja apresenta maior taxa de deterioração do que a de milho.

2. A taxa de deterioração da semente $(\operatorname{tg} \beta)$ é influenciada pela temperatura e umidade relativa do ambiente e é específica para cada espécie.

3. A equação simplificada de viabilidade $\mathrm{Vt}=\mathrm{Vi}$ $(\operatorname{tg} \beta)$. p mostra-se eficaz e promissora na predição da germinação de sementes de milho e soja em armazenamento aberto.

\section{Agradecimentos}

À equipe do Laboratório de Sementes da Embrapa Milho e Sorgo, Sete Lagoas, MG, pela ajuda na execução deste trabalho.

\section{Referências}

BRASIL. Ministério da Agricultura e Reforma Agrária. Regras para análise de sementes. Brasília, 1992. 365p.

BURRIS, J.S. Maintenance of soybean seed quality in storage as influenced by moisture, temperature and genotype. Iowa State Journal of Research, v.54, p.377-389, 1980.

DELOUCHE, J.C.; BASKIN, C.C. Accelerated aging techniques for 
predicting the relative storability of seed lots. Seed Science and Technology, v.1, p.427-452, 1973

ELLIS, R.H.; HONG, T.D.; ROBERTS, E.H.; TAO, K.L. LOW moisture content limits to relations between seed longevity and moisture. Annals of Botany, v.65, p.493-504, 1990.

ELLIS, R.H.; ROBERTS, E.H. Improved equations for the prediction of seed longevity. Annals of Botany, v.45, p.13-30, 1980

FINNEY, D.J. Probit analysis. $3^{\text {rd }}$ ed. London: Cambridge University Press, 1971. 318p.

ROBERTS, E.H. Predicting the storage life of seeds. Seed Science and Technology, v.1, p.499-514, 1973

ROBERTS, E.H. Quantifying seed deterioration. In: McDONALD, M.B.; NELSON, C.J. (Ed.). Physiology of seed deterioration. Madison: Crop Science Society of America, 1986. p.101-123. (Special Publication, 11).

ROBERTS, E.H. Storage environment and the control of viability. In: ROBERTS, E.H. (Ed.). Viability of seeds. New York: Syracuse University Press, 1972. p.14-58.
ROBERTS, E.H. The viability of cereal seed in relation to temperature and moisture. Annals of Botany, v.24, p.12-31, 1960.

ROBERTS, E.H. The viability of rice seed in relation to temperature, moisture content and gaseous environment. Annals of Botany, v.25, p.381-390, 1961

ROBERTS, E.H.; ABDALLA, F.H. The influence of temperature, moisture, and oxygen on period of seed viability in barley, broad beans and peas. Annals of Botany, v.32, p.97-117, 1968.

ROBERTS, E.H.; ELLIS, R.H. Water and seed survival. Annals of Botany, v.63, p.39-52, 1989

TEKRONY, D.M.; NELSON, C.; EGLI, D.B.; EGLI, G.M. Predicting soybean seed deterioration during warehouse storage. Seed Science and Technology, v.21, p.127-137, 1993.

VERTUCCI, C.W.; ROOS, E.E. Seed storage temperature and relative humidity (correspondence). Seed Science Research, v.3, p.215 216, 1993.

VERTUCCI, C.W.; ROOS, E.E. Theoretical basis of protocols for seed storage. Plant Physiology, v.94, p.1019-1023, 1990

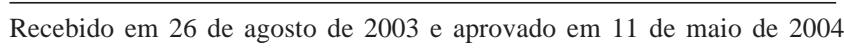

